\title{
Activation of Autophagy and Nrf2 Signaling in Human Breast Adenocarcinoma MCF-7 Cells by Novel Monophenolic Antioxidants
}

\author{
E. B. Menshchikova ${ }^{a, *}$, A. V. Chechushkov ${ }^{a}$, P. M. Kozhin ${ }^{a}$, S. V. Kholshin ${ }^{b}$, N. V. Kandalintseva ${ }^{b}$, \\ G. G. Martinovich ${ }^{c}$, and N. K. Zenkov ${ }^{a}$ \\ ${ }^{a}$ Federal Research Center for Fundamental and Translational Medicine, Novosibirsk, 630117 Russia \\ ${ }^{b}$ Novosibirsk State Pedagogical University, Novosibirsk, 630126 Russia \\ ${ }^{c}$ Belarusian State University, Minsk, 220030 Belarus \\ *e-mail:lemen@centercem.ru
}

Received June 26, 2018; revised July 26, 2018; accepted July 30, 2018

\begin{abstract}
The effect of novel water-soluble structurally related monophenolic compounds on the activity of two most important mechanisms of maintaining intracellular homeostasis, autophagy and the redox-sensitive signal system Keap1/Nrf2/ARE, has been studied in human breast adenocarcinoma cell line MCF-7 using confocal microscopy. Autophagy processes were analyzed on the basis of the amount of intracellular vesicles that were positive for the autophagy marker (LC3B). The activation of the Keap1/Nrf2/ARE system was determined by the translocation of the transcription factor Nrf2 into the nucleus. It was found that the effect of the tested compounds depended on their structure and concentration. When the inhibitor of autophagosome-lysosome fusion chloroquine was added to the culture medium $(20 \mu \mathrm{M})$, the asymmetrically hindered by the tert-butyl group phenols with thiosulfonate (TS-13) and sulfonate group in the para-propyl substituent increased the rate of autophagosome elimination in MCF-7 cells. Shortening of the para-alkyl substituent by one methylene unit abolished the effect. The addition of the second ortho-tert-butyl substituent had the reverse result. Both tested compounds enhanced the translocation of the transcription factor Nrf2 into the nucleus of MCF-7 cells (which is a critical step in Keap1/Nrf2/ARE activation). It was observed after incubation with asymmetrically hindered by the tert-butyl group phenol with selenosulfonate group in parapropyl substituent $(5-100 \mu \mathrm{M})$ for $4 \mathrm{~h}$ and with TS-13 $(5-100 \mu \mathrm{M})$ for $24 \mathrm{~h}$. Taking into account our previous findings on the toxicity of this group of compounds for MCF-7 cells we can conclude that these compounds exert different effect on autophagy and activation of the antioxidant response element signaling system Keap1/Nrf2/ARE.
\end{abstract}

Keywords: MCF-7 cell line, Keap1/Nrf2/ARE system, nuclear-cytoplasmic ratio, autophagosome, LC3B, chloroquine, synthetic monophenols

DOI: $10.1134 / \mathrm{S} 1990519 X 1902007 \mathrm{X}$

\section{INTRODUCTION}

Radical $\left(\mathrm{O}_{2}^{-}, \mathrm{NO}^{*}, \mathrm{RO}^{*}\right)$ and nonradical $\left(\mathrm{H}_{2} \mathrm{O}_{2}\right.$, $\mathrm{ONOOH}, \mathrm{ROOH}$ ) active oxygen (ROS) and nitrogen species are key regulators of biological processes. However, their activity often becomes toxic and destructive, especially in pathological processes. The

\footnotetext{
Abbreviations: ROS-reactive oxygen species; TS-12-sodium 3-(3'-tert-butyl-4'-hydroxyphenyl)ethyl thiosulfonate; TS-13sodium 3-(3'-tert-butyl-4'-hydroxyphenyl)propyl thiosulfonate; TS-17-sodium 3-(3',5'-di-tert-butyl-4'-hydroxyphenyl)propyl thiosulfonate; S-13-sodium 3-(3'-tert-butyl-4'-hydroxyphenyl)propyl sulfonate; SeS-13-sodium 3-(3'-tert-butyl-4'hydroxyphenyl)propyl selenosulfonate; $\mathrm{NC}$-nuclear-cytoplasmic ratio; ARE-antioxidant response element; FBS-fetal bovine serum; LC3B-microtubule-associated proteins 1A/1B light chain; Nrf2-NF-E2 related factor; Keap1-Kelch-like ECH-associated protein 1; p62/SQSTM-ubiquitin-binding protein p62, also known as sequestosome 1.
}

phenomenon is known as "oxidative stress" (Niki, 2016; Sies et al., 2017; Chikara et al., 2018; Kapuy et al., 2018). A low level of ROS in cells is maintained with a multilevel system of enzymatic (superoxide dismutase, catalase, glutathione peroxidase, etc.) and nonenzymatic (glutathione and other $\mathrm{SH}$-containing compounds, phenols) antioxidants. For a long time, it has been thought that the antioxidant activity of phenols has an antiradical effect and that they act as chelators of transition metal ions, interrupting the chain processes of free radical oxidation (Menshchikova et al., 2012). In recent years, the mechanisms of indirect phenol protection during oxidative stress, namely, their ability to induce autophagy and activate the signal system of the antioxidant response element Keap1/Nrf2/ARE, have been actively studied (Zen- 


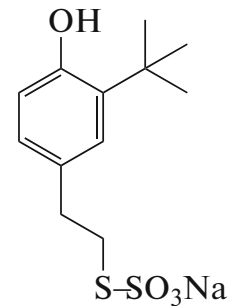

TS-12

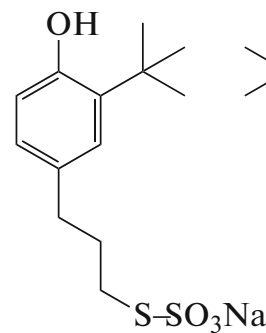

TS-13

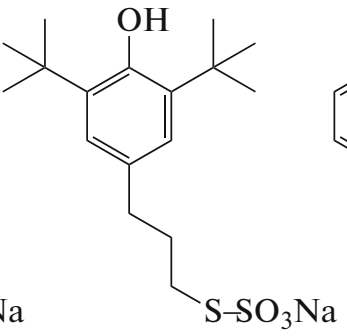

TS-17

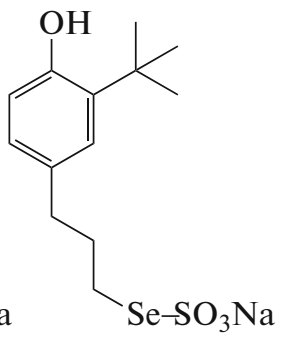

SeS-13

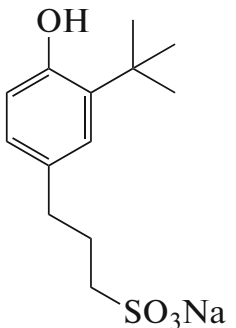

S-13

Fig. 1. Structural formulas of applied synthetic monophenols. For explanations, see MATERIALS AND METHODS.

kov et al., 2016; Nabavi et al., 2017; Shabalala et al., 2017; Zhao et al., 2018).

Autophagy, being the main mechanism of degradation of intracellular components (proteins, nucleic acids, fat accumulations, organelles), is very important for protection from oxidative and carbonyl stress. It removes damaged mitochondria and peroxisomes (intracellular sources of ROS), as well as protein and lipid aggregates, which are a cause of carbonyl compound toxicity (Fedorova et al., 2014; Giordano et al., 2014).

More than 20 redox-sensitive transcription factors are currently known. Nrf2, which regulates the expression of genes with the antioxidant response element ARE, is of particular importance. In cells, Nrf2 is under control of the repressor protein Keap1, which is a molecular sensor of changes in the intracellular redox balance. The function of the Keap1/Nrf2/ARE redox-sensitive signaling system is to maintain internal homeostasis under apoptosis-inducing, carcinogenic, and stressful conditions. ARE controls the expression of more than 500 genes. They include two large groups of antioxidant enzymes and phase II xenobiotic detoxification enzymes (Turpaev, 2013). The biological function of the Keap1/Nrf2/ARE system regulating the intracellular redox balance is to control the activity of a wide range of redox-sensitive transcription factors, as well as metabolic processes involving phosphatases and kinases. An increased activity of Nrf2-mediated processes enhances autophagy caused by degradation of Keap1 with the involvement of the autophagy adapter protein of p62/SQSTM (Zenkov et al., 2017) and ARE elements in promoters of many autophagy genes (Pajares et al., 2017). In recent years, there has been an active search for new activators and inhibitors of Nrf2 transcriptional activity and autophagy to prevent and treat a wide range of diseases (Zenkov et al., 2013; Chu et al., 2017; Gazaryan and Thomas, 2016).

The study and practical application of natural phenols is largely hindered by their hydrophobicity and the complexity of the molecule structural organization. Previously performed studies allowed us to reveal the relationship between the structure of structurally related novel hydrophilic monophenols and their antioxidant and biological activity in vitro and in vivo. It was found that the substances not only directly inhibit free radical oxidative processes involving ROS, but also suppress the development of oxidative stress indirectly through the activation of intracellular defense systems.

The purpose of this study was to investigate the relationship between the structure of new synthetic monophenolic antioxidants and their effect on the autophagy activity and Nrf2-mediated processes.

\section{MATERIALS AND METHODS}

Five novel structurally interrelated hydrophilic phenolic compounds were synthesized: sodium 3-(3'-tertbutyl-4'-hydroxyphenyl)ethyl thiosulfonate (TS-12), 3-(3'-tert-butyl-4'-hydroxyphenyl)propyl thiosulfonate (TS-13), sodium 3-(3',5'-di-tert-butyl-4'-hydroxyphenyl)propyl thiosulfonate (TS-17), sodium 3-(3'-tertbutyl-4'-hydroxyphenyl)propyl sulfonate (S-13), sodium 3-(3'-tert-butyl-4'-hydroxyphenyl)propyl selenosulfonate $(\mathrm{SeS}-13)$. Their structure formulas are shown in Fig. 1. Compounds were obtained from 2,6-di-tertbutylphenol according to the sequence of transformations described previously (Oleinik et al., 2007; Gainutdinov et al., 2018). Their structure was confirmed by elemental analysis and NMR, IR, and UV spectroscopy.

Experiments were performed with MCF-7 human breast adenocarcinoma cells. The cells were cultured in DMEM medium containing $10 \%$ fetal bovine serum (FBS), $1 \%$ penicillin, $1 \%$ streptomycin, and $1 \%$ glutamine. Cells that reached $90 \%$ confluence were detached by pipetting and seeded in a 24-well plate $\left(25 \times 10^{3}\right.$ cells/well). Tested compounds were added after $24 \mathrm{~h}$. Each compound was diluted in prewarmed sterile deionized water (miliQ quality) and aliquoted. Each aliquot was 200 times the stock solution for each 


\section{$I$}

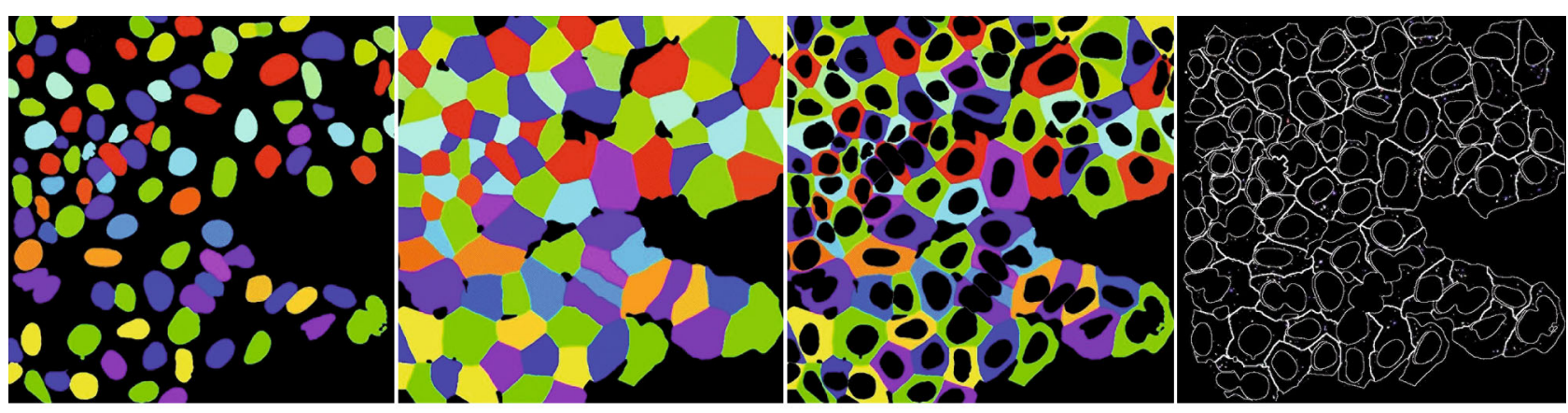

II
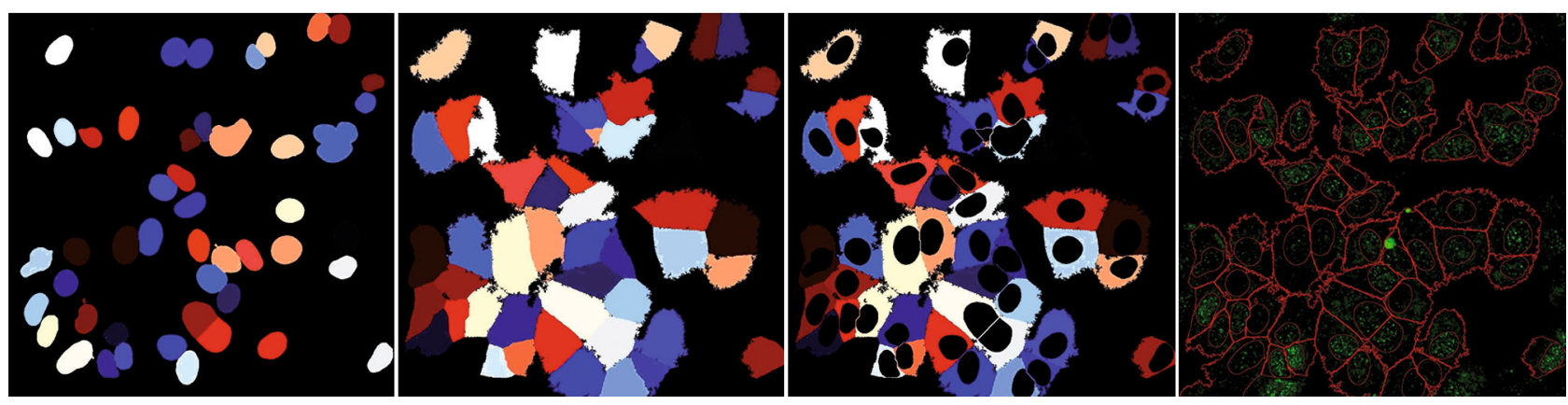

Fig. 2. Sequence of cell segmentation for the study of $(I)$ autophagy and (II) Nrf2 nuclear-cytoplasmic ratio. (a) Nucleus segmentation, (b) segmentation of cytoplasm around the nucleus, (c) cytoplasm assigment, and (d) identification of vesicles $(I)$ the cytoplasm or $(I I)$ overlaying the cytoplasm contour on the original image. Pseudocolor is put on fragments $(\mathrm{a}-\mathrm{c})$ for convenience of perception of individual cells.

concentration. Aliquots were added in tubes with culture medium and kept for $5 \mathrm{~min}$ at $37^{\circ} \mathrm{C}$. The medium in cell culture was replaced with these solutions and cells were cultivated for $24 \mathrm{~h}$. To control autophagosome formation and lysosomal degradation, $60 \mu \mathrm{M}$ chloroquine, an autophagosome-lysosome fusion blocker (C6628, Sigma, United States), was introduced into the culture medium $23 \mathrm{~h}$ after the test compound was added and cultured for $1 \mathrm{~h}$.

Twenty-four hours after the addition of tested compounds the medium was discarded. The cells were fixed with $10 \%$ formalin, permeabilized in $0.15 \%$ Triton X-100, and incubated with blocking solution (PBS with $5 \%$ bovine serum albumin (BSA), $0.05 \%$ Triton $\mathrm{X}-100)$ on a plate shaker-thermostat for $40 \mathrm{~min}$ at $37^{\circ} \mathrm{C}$. Cells were incubated $\left(60 \mathrm{~min}, 37^{\circ} \mathrm{C}\right)$ with primary rabbit antibodies to mammalian LC3B protein (L10382, Invitrogen, United States) added to the BSA-free wash solution. Samples were washed five times with BSA-free solution and incubated with Alexa Fluor 488 conjugated antirabbit antibodies (Abcam, United States) for $30 \mathrm{~min}$ at $37^{\circ} \mathrm{C}$. PBS was removed by short washing with deionized water and cells were mounted in FluoroShield mounting medium (Abcam, United States) containing DAPI for nuclei staining. Intracellular LC3B localization was examined under an LSM 710 confocal laser scanning microscope (Zeiss AG, Germany) using a Plan-Apochromat $63 \times / 1.4$ objective.

Images were analyzed with the CellProfiler program (http://cellprofiler.org). For the computer analysis the nuclei were segmented for DAPI marker (Fig. 2Ia). The total LC3B signal (images of the corresponding fluorescence channel were transformed with Gaussian filter) associated with each nucleus was then segmented for cell assignment (Fig. 2/b). Each entity was then divided into cytoplasmic and nuclear compartments. The resulting cytoplasm contours were superimposed on the original image in order to segment the vesicles and their associations with cytoplasmic compartments (Fig. 2Id). The total and average fluorescence intensity of fluorochrome reflecting the amount of LC3B protein was determined for cytoplasmic compartments.

The capacity of synthesized compounds to activate Keap1/Nrf2/ARE redox-sensitive signaling system was assayed by translocation of Nrf2 factor into the nucleus (Zenkov et al., 2017). TS-13 and SeS-13 
exhibited the maximum and minimum toxicity for human tumor cells, respectively (Gainutdinov et al., 2017) were used as control. MCF-7 cells were treated with tested compounds under the conditions described above. The cells were exposed to these drugs for 4 and $24 \mathrm{~h}$. Cells were incubated with primary rabbit antibodies to Nrf2 (ab31163, Abcam, United Kingdom) for $1 \mathrm{~h}$ at $37^{\circ} \mathrm{C}$. The cells were washed three times, treated with Alexa Fluor 488-conjugated secondary antibodies $\left(30 \mathrm{~min}, 37^{\circ} \mathrm{C}\right)$, and, after washing, were mounted in the mounting medium Fluoroshield containing DAPI dye. Intracellular Nrf2 localization was examined under an LSM 710 the confocal laser scanning microscope.

Images were analyzed with the CellProfiler program as described above. For computer analysis, nuclei were segmented for DAPI marker (Fig. 2IIa). The total Nrf2 signal associated with each nucleus was segmented for cell assignment (Fig. 2I/b). Then each entity was divided into cytoplasmic and nuclear compartments (Fig. 2IIc). The resulting cytoplasm contours were superimposed on the original image (Fig. 2IId). The total and average Alexa Fluor 488 fluorescence intensity in each compartment gave the information on the amount of Nrf2. All segmented cells of each group were combined into one cell population to determine the fluorescence intensity in the nucleus and cytoplasm and its nuclear-cytoplasmic ratio.

Quantitative data was preliminary assessed for normal distribution according to the Shapiro-Wilk test. Continuous variables are represented in the normal distribution as an arithmetic mean and standard deviation and as median and interquartile intervals in other than the normal distribution. Mann-Whitney and Dunn criteria were used to assess the differences in quantitative data. The significance level of the null statistical hypothesis (p) was 0.05 .

\section{RESULTS AND DISCUSSION}

Autophagy occurrence was assessed by visualization of autophagosome and intracellular vesicles positive for the LC3B marker. It should be noted that a number of vesicular structures of varying intensity and size are normally observed in MCF-7 cells. Since the size of most autophagosomes is actually below the limit of an optical microscope's resolution (except for SuperResolution class systems), the only objective criterion for identification an intracellular object as an autophagosome is the intensity of the vesicle fluorescent signal.

All experiments were performed with and without chloroquine. The gold standard in determining the autophagic activity is the registration of the auto- phagic flux. LC3B-positive autophagosomes are constantly degraded in lysosomes. Chloroquine addition blocks the autophagosome-lysosome fusion. The amount of autophagosomes observed reflects the intensity of their formation. The ratio of the autophagosome number in cultures with and without chloroquine (coefficient $k_{\mathrm{CQ}}$ ) shows the ability of cells to remove newly formed autophagosomes (Klionsky et al., 2016) and is an important indication of the protective properties of autophagy.

The selenium-containing analogue SeS-13 exhibited high toxicity. MCF-7 cells incubated with this drug for $24 \mathrm{~h}$ lost the morphological features of adhesive cells. Analysis of intracellular formations and distribution of autophagosomes (LC3B-positive vesicles) was impossible; therefore, the data on this compound were not presented. A typical difference in the amount of detected autophagosomes was observed in control cells exposed and not exposed to chloroquine (Fig. 3a). The studied compounds, with the exception of TS-13 $(5 \mu \mathrm{M})$, slightly reduced the rate of new autophagosome formation (group with chloroquine, Fig. 3a) and removal of produced autophagosome (coefficient $k_{\mathrm{CQ}}$, Fig. 3b). At a higher concentration of compounds $(20 \mu \mathrm{M})$, these processes steadily enhanced and became close to control (TS-12) or exceeded it (TS-13, S-13). TS-17 compound (fully hindered monophenol) exhibited low rates of autophagosome removal visible as a decreased $k_{\mathrm{CQ}}$ coefficient.

The study the monophenols effect on the activity of the Nrf2-dependent signaling pathway demonstrated that after $4 \mathrm{~h}$ of incubation, SeS-13 facilitated the dose-dependent increase in the Nrf2 content in the nucleus of MCF-7 cells (Fig. 4a). At the concentration of $100 \mu \mathrm{M}$, the nuclear translocation of the transcription factor increased (Fig. 4b). MCF-7 cells exposed to $\mathrm{SeS}-13$ for $24 \mathrm{~h}$, as mentioned above, lost adhesive cell morphology and it was impossible to assay the Nrf2 intracellular content and distribution. Supposedly, the drug toxicity induced the homeostatic Keap1/Nrf2/ARE system. When cells were cultured in the presence of TS-13, the total analysis of the cell population cultivated with TS-13 revealed that the monophenol initially reduced the nuclear-cytoplasmic (NC) ratio without affecting the Nrf2 content in the nucleus $(4 \mathrm{~h})$ and then $(24 \mathrm{~h})$ increased both NC and Nrf2 amount in the nucleus (Fig. 4). It was found that the distribution of fluorescence in the total cell population (Figs. 5a, 5b) differed from the distribution of average fluorescence for individual fields of view (Figs. 5c, 5d). In the first case, the distribution was more consolidated, while, in the second case, the cells were distinguished in $\mathrm{NC}$ ratio of the Nrf 2 content. In cell cultures incubated with presence of $20 \mu \mathrm{M}$ TS-13 


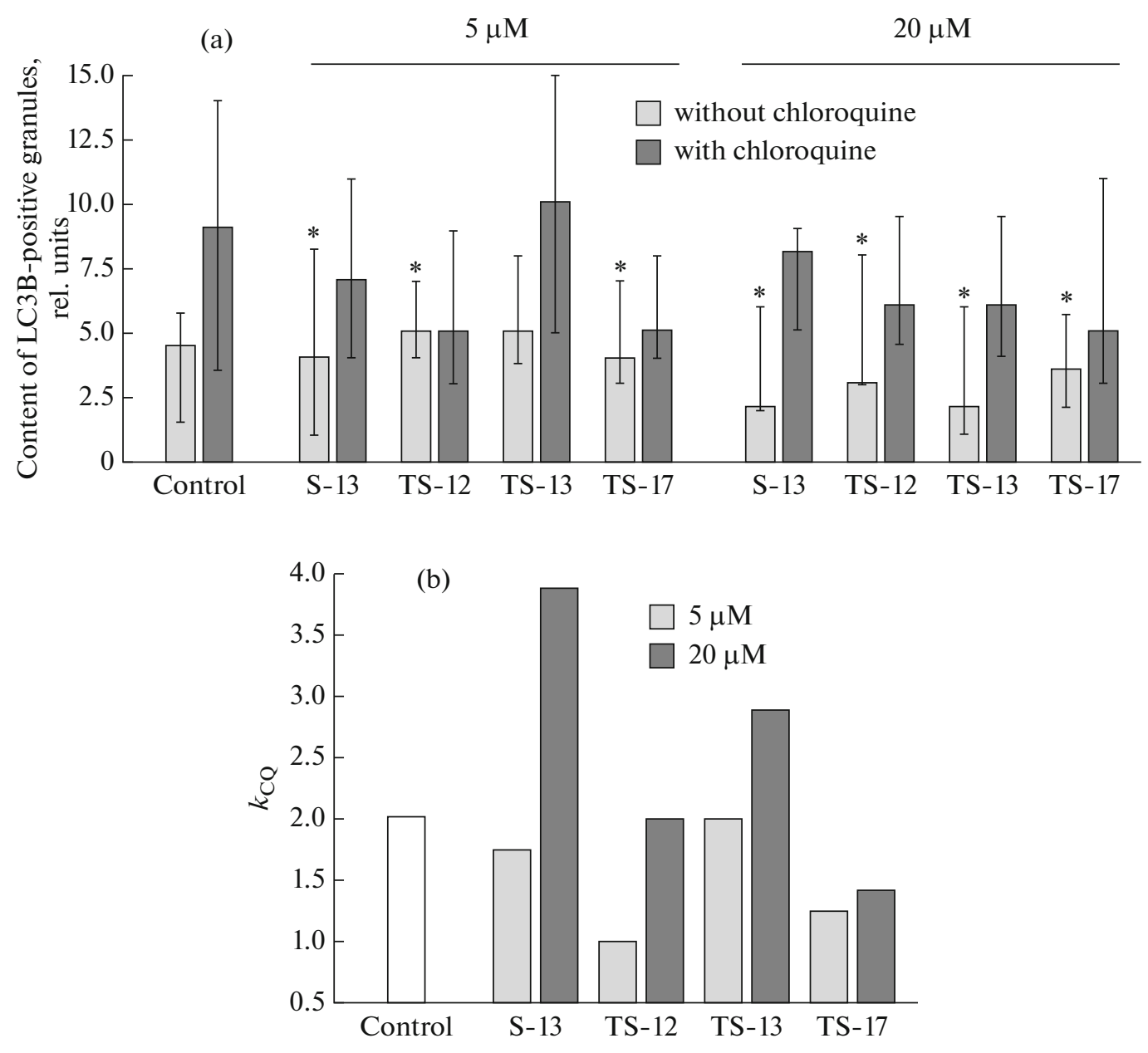

Fig. 3. (a) Amount of LC3B-positive autophagosome vesicles and (b) coefficient $k_{\mathrm{CO}}$ in MCF-7 cells exposed to monophenols with or without chloroquine $(1 \mathrm{~h})$. Median values and interquartile ranges are presented. Asterix shows significant difference from control $(\mathrm{p}<0.05)$.

a relatively small pool of cells responded by a drastic increase (more than three times) in the value of the index, while the data summing and averaging indicated its seeming decrease as described above.

Many plant phenols display antitumor activity with a multiplicity of mechanisms action. The role of autophagy in carcinogenesis and cancer therapy is complex and depends on the stage of the process, tumor cell microenvironment, treatment scenario. On the one hand, irreversible self-destruction caused by massive autophagy leads to the death of malignant cells (Klimaszewska-Wisniewska et al., 2016). On the other hand, inhibition of autophagy as a protective mechanism of tumor cells allows their chemoresistance to be overcome (Wang et al., 2018). In this regard, it is significant that TS-17, which exhibited the highest toxicity of the four tested compounds, inhibited both the formation rate and the rate of autophagosome removal in MCF-7 cells. Other drugs at a concentration of
$20 \mu \mathrm{M}$ increased autophagy ( $k_{\mathrm{CQ}}$ coefficient) reciprocally to their toxicity (Gainutdinov et al., 2018). Supposedly, the stronger the cell responds to the compound by induction of autophagy (the mechanism of self-defense), the less pronounced is antitumor effect (Lee et al., 2016). This may be due to the paradoxical prooxidant effect of these compounds, which is indirectly confirmed by the inverse relationship between their antioxidant activity in the cell-free system and toxicity to MCF-7 cells (Gainutdinov et al., 2018).

A key mechanism of the antitumor activity of phenolic compounds is their ability to activate the Keap1/Nrf2/ARE system (Rajagopal et al., 2018). Networks of transcriptional programs controlled by $\mathrm{Nrf} 2$ (mainly antioxidant and detoxification) allow the cell to adapt and survive in various stressful conditions, which, along with its anti-inflammatory effect and influence on mitochondrial function and intermediate metabolism, underlies the chemopreventive 

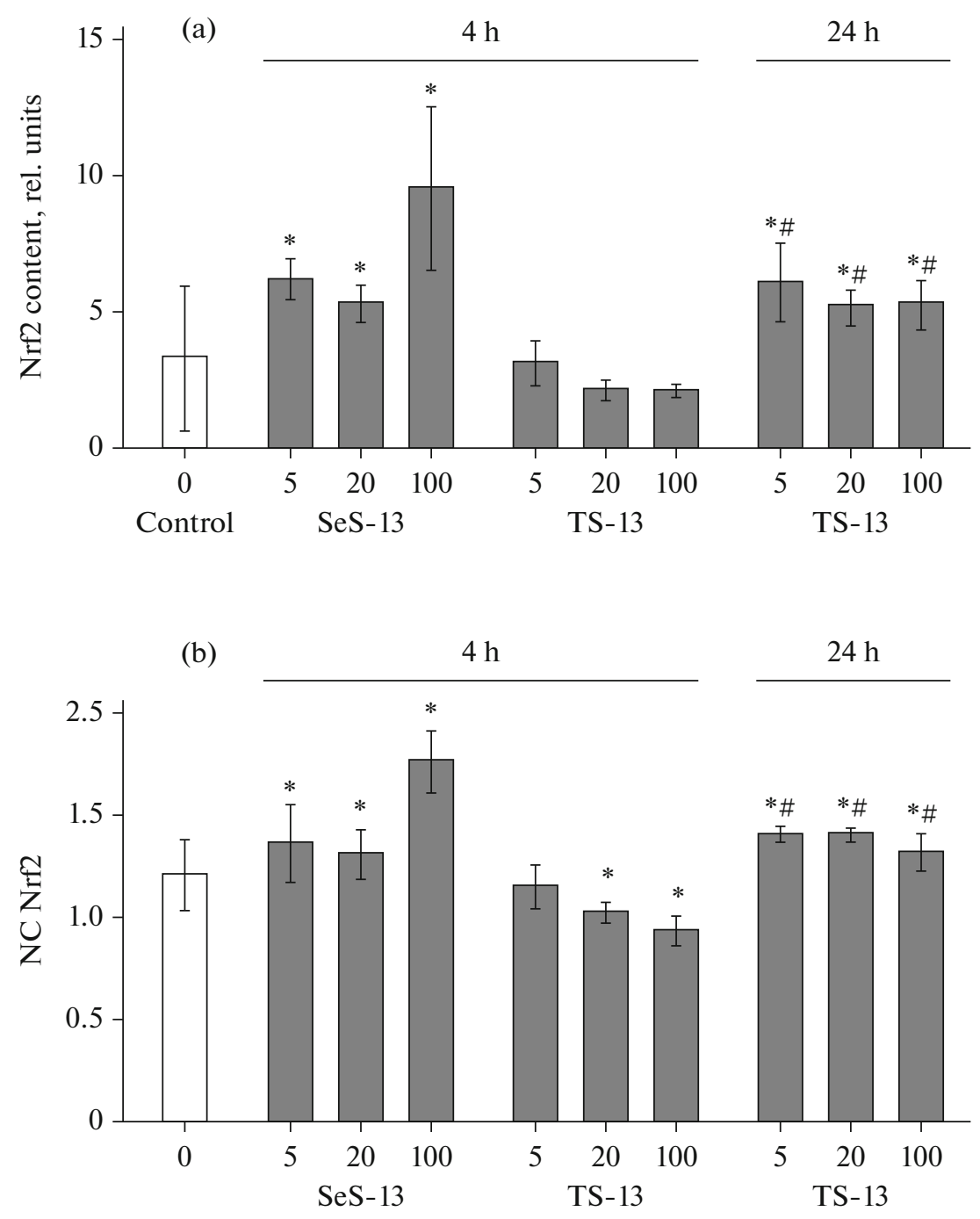

Fig. 4. (a) Nrf2 content in nucleus and (b) nuclear-cytoplasmic ratio (NC) in MCF-7 cells exposed to TS-13 and SeS-13 monophenols $(5,20,100 \mu \mathrm{M})$ for 4 and $24 \mathrm{~h}$. Median values and interquartile ranges are presented. Significant difference from control is shown by * and \# from $4 \mathrm{~h}$ of incubation $(p<0.05)$. Analysis of Nrf2 intracellular content and distribution in MCF-7 cells cultivated with SeS-13 for $24 \mathrm{~h}$ was not possible (see the text).

effects of Nrf2, the ability to suppress carcinogenesis at the promotion stage (Dinkova-Kostova et al., 2017). At the same time, the cells of already formed tumor are able to effectively use Nrf2, rightly called a "two-faced Janus," for their own protection. Its high activity in different malignant tumors gives a poor prognosis of therapy, correlating with a decreased life expectancy of patients (Kitamura and Motohashi, 2018).

We are inclined to suggest that the induction of the Keap1/Nrf2/ARE system in MCF-7 cells in response to the two tested synthetic monophenols under these conditions is not so much due to their direct ability to increase the stability of the transcription factor Nrf2 as it is a manifestation of the self-defense of tumor cells in response to reduction of redox buffer capacity
(Martinovich et al., 2017), as well as increased autophagy (Kapuy et al., 2018).

Thus, it can be concluded that the ability of novel water-soluble monophenolic antioxidants, distinguished by a number of tert-butyl substituents and the structure of the para-alkyl substituent, to inhibit the viability of MCF-7 tumor cells (Gainutdinov et al., 2018 ) is accompanied by activation of the antioxidant response element signal system Keap1/Nrf2/ARE and autophagy. The effect depends on the structure and concentration of the compound.

\section{ACKNOWLEDGMENTS}

This study was supported by the Russian Foundation for Basic Research (project no. 16-54-00050-Bel- 
(a)

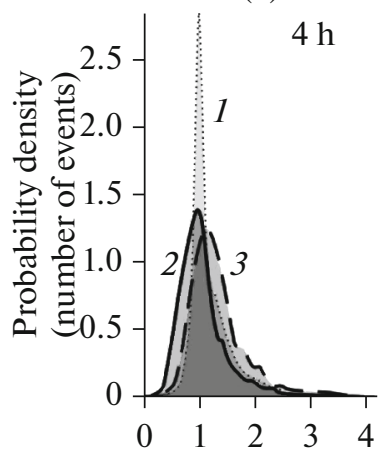

(b)

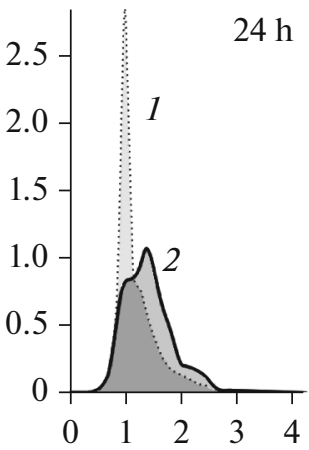

(c)

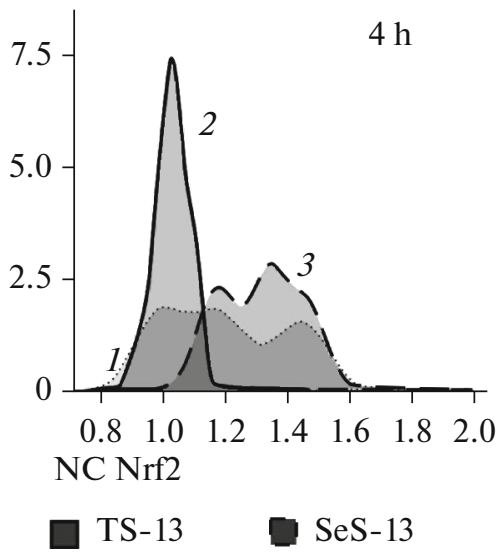

(d)

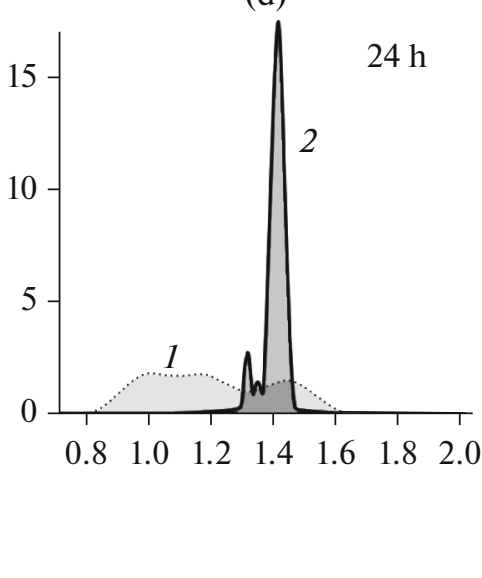

Fig. 5. Distribution of $(a, b)$ fluorescence of the total cell population and $(c, d)$ its average values in single fields of view depending on the nuclear-cytoplasmic (NC) ratio of the Nrf2 content in MCF-7 cells (1) in the control and exposed for different times to monophenols (2) TS-13 and (3) SeS-13 at concentrations of $20 \mu \mathrm{M}$.

a) and Belarusian Republican Foundation for Fundamental Research (project M16P-022). Equipment from the Modern Optical Systems Center for Collective Use was used.

\section{COMPLIANCE WITH ETHICAL STANDARDS}

The authors declare that they have no conflict of interest. This article does not contain any studies involving animals or human participants performed by any of the authors.

\section{REFERENCES}

Chikara, S., Nagaprashantha, L.D., Singhal, J., Horne, D., Awasthi, S., and Singhal, S.S., Oxidative stress and dietary phytochemicals: role in cancer chemoprevention and treatment, Cancer Lett., 2018, vol. 413, pp. 122-134.

Chu, X.Y., Liu, Y.M., and Zhang, H.Y., Activating or inhibiting Nrf2?, Trends Pharmacol. Sci., 2017, vol. 38, pp. 953-955.

Dinkova-Kostova, A.T., Fahey, J.W., Kostov, R.V., and Kensler, T.W., KEAP1 and done? Targeting the NRF2 pathway with sulforaphane, Trends Food Sci. Technol., 2017, vol. 69, pp. 257-269.

Fedorova, M., Bollineni, R.C., and Hoffmann, R., Protein carbonylation as a major hallmark of oxidative damage: update of analytical strategies, Mass Spectrom. Rev., 2014, vol. 33, pp. 79-97.

Gainutdinov, P.I., Kozhin, P.M., Chechushkov, A.V., Martinovich, G.G., Kholshin, S.V., Kandalintseva, N.V., Zenkov, N.K., and Menshchikova, E.B., Inverse relationship between the antioxidant activity of structurally related synthetic monophenols and their toxicity in tumor cells, Sib. Nauch. Med. Zh., 2018, vol. 38, no. 1, pp. 22-31.

Gazaryan, I.G. and Thomas, B., The status of Nrf2-based therapeutics: current perspectives and future prospects, Neural Regenerat. Res., 2016, vol. 11, pp. 1708-1711.
Giordano, S., Darley-Usmar, V., and Zhang, J., Autophagy as an essential cellular antioxidant pathway in neurodegenerative disease, Redox Biol., 2014, vol. 2, pp. 82-90.

Kapuy, O., Papp, D., Vellai, T., Banhegyi, G., and Korcsmaros, T., Systems-level feedbacks of NRF2 controlling autophagy upon oxidative stress response, Antioxidants (Basel), 2018, vol. 7, no. 3, p. E39.

Kitamura, H. and Motohashi, H., NRF2 addiction in cancer cells, Cancer Sci., 2018, vol. 109, pp. 900-911.

Klimaszewska-Wisniewska, A., Halas-Wisniewska, M., Tadrowski, T., Gagat, M., Grzanka, D., and Grzanka, A., Paclitaxel and the dietary flavonoid fisetin: a synergistic combination that induces mitotic catastrophe and autophagic cell death in A549 non-small cell lung cancer cells, Cancer Cell Int., 2016, vol. 16, pp. 1-23.

Klionsky, D.J., Abdelmohsen, K., Abe, A., Abedin, M.J., et al., Guidelines for the Use and Interpretation of Assays for Monitoring Autophagy (3rd ed.), Autophagy, 2016, vol. 12, pp. 1-222.

Lee, Y.J., Lee, G.J., Yi, S.S., Heo, S.H., Park, C.R., Nam, H.S., Cho, M.K., and Lee, S.H., Cisplatin and resveratrol induce apoptosis and autophagy following oxidative stress in malignant mesothelioma cells, Food Chem. Toxicol., 2016, vol. 97, pp. 96-107.

Martinovich, G.G., Martinovich, I.V., Vcherashniaya, A.V., Zenkov, N.K., Menshchikova, E.B., Kandalintseva, N.V., and Cherenkevich, S.N., Mechanisms of redox regulation of chemoresistance in tumor cells by phenolic antioxidants, Biophysics (Moscow), 2017, vol. 62, no. 6, pp. 942-949.

Menshchikova, E.B., Lankin, V.Z., and Kandalintseva, N.V., Fenol'nye antioksidanty $v$ biologii i meditsine (Phenolic Antioxidants in Biology and Medicine), Saarbrücken: LAP LAMBERT Acad. Publ., 2012.

Nabavi, S.F., Sureda, A., Dehpour, A.R., Shirooie, S., Silva, A.S., Devi, K.P., Ahmed, T., Ishaq, N., Hashim, R., Sobarzo-Sanchez, E., Daglia, M., Braidy, N., Volpicella, M., Vacca, R.A., and Nabavi, S.M., Regulation of autophagy by polyphenols: paving the road for treatment 
of neurodegeneration, Biotechnol. Adv., 2017. doi 10.1016/j.biotechadv.2017.12.001

Niki, E., Oxidative stress and antioxidants: distress or eustress?, Arch. Biochem. Biophys., 2016, vol. 595, pp. 19-24.

Oleinik, A.S., Kuprina, T.S., Pevneva, N.Yu., Markov, A.F., Kandalintseva, N.V., Prosenko, A.E., and Grigoriev, I.A., Synthesis and antioxidant properties of sodium S-[3-(hydroxyaryl)propyl] thiosulphates and [3(hydroxyaryl)propane]-1-sulfonates, Izv. Akad. Nauk, Ser. Khim., 2007, vol., no. 6, pp. 1094-1101.

Pajares, M., Cuadrado, A., and Rojo, A.I., Modulation of proteostasis by transcription factor NRF2 and impact in neurodegenerative diseases, Redox Biol., 2017, vol. 11, pp. 543-553.

Rajagopal, C., Lankadasari, M.B., Aranjani, J.M., and Harikumar, K.B., Targeting oncogenic transcription factors by polyphenols: a novel approach for cancer therapy, Pharmacol. Res., 2018, vol. 130, pp. 273-291.

Shabalala, S., Muller, C.J.F., Louw, J., and Johnson, R., Polyphenols, autophagy and doxorubicin-induced cardiotoxicity, Life Sci., 2017, vol. 180, pp. 160-170.

Sies, H., Berndt, C., and Jones, D.P., Oxidative stress, Annu. Rev. Biochem., 2017, vol. 86, pp. 715-748.

Turpaev, K.T., Keap1-Nrf2 signaling pathway: mechanisms of regulation and role in protection of cells against toxicity caused by xenobiotics and electrophiles, Biochemistry (Moscow), 2013, vol. 78, no. 2, pp. 111-126.

Wang, W., Chen, D., and Zhu, K., SOX2OT variant 7 contributes to the synergistic interaction between EGCG and doxorubicin to kill osteosarcoma via autophagy and stemness inhibition, J. Exp. Clin. Cancer Res., 2018, vol. 37, p. 37.

Zenkov, N.K., Menshchikova, E.B., and Tkachev, V.O., Keap1/Nrf2/ARE redox-sensitive signaling system as a pharmacological target, Biochemistry (Moscow), 2013, vol. 78, no. 1, pp. 19-36.

Zenkov, N.K., Chechushkov, A.V., Kozhin, P.M., Kandalintseva, N.V., Martinovich, G.G., and Menshchikova, E.B., Plant phenols and autophagy, Biochemistry (Moscow), 2016, vol. 81, no. 4, pp. 297-314.

Zenkov, N.K., Kozhin, P.M., Chechushkov, A.V., Martinovich, G.G., Kandalintseva, N.V., and Menshchikova, E.B., Mazes of Nrf2 regulation, Biochemistry (Moscow), 2017, vol. 82, no. 5, pp. 556-564.

Zhao, Y., Song, W., Wang, Z., Jin, X., Xu, J., Bai, L., Li, Y., Cui, J., and Cai, L., Resveratrol attenuates testicular apoptosis in type 1 diabetic mice: role of Akt-mediated Nrf2 activation and P62-dependent Keap1 degradation, Redox Biol., 2018, vol. 14, pp. 609-617.

Translated by I. Fridlyanskaya 